\section{Encouraging good oral care habits}

When illness strikes, you can encourage patients to continue practising good oral care habits by offering reliable solutions from Curaprox.

Among the product offerings are the Be You range of toothpastes - available in six unique flavours - and the colourful CS 5460 manual toothbrush, featuring an octagonal handle and a compact brush head with 5,460 ultra-fine CUREN filaments.

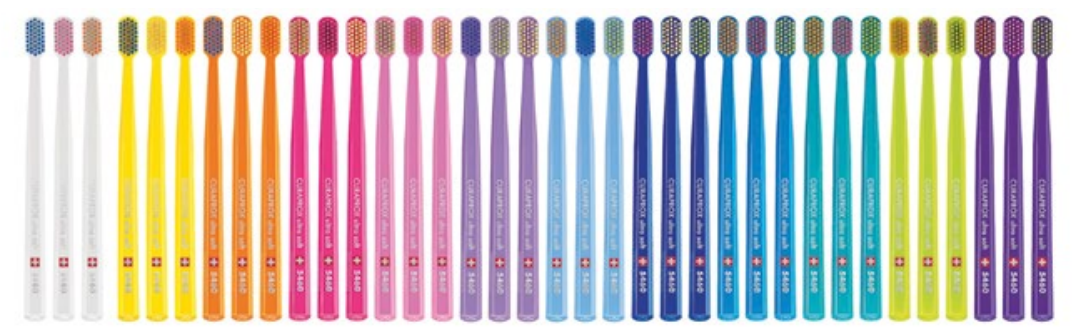

\section{Increasing sustainability}

Leading oral hygiene brand, TePe, has added more options to its sustainably produced TePe GOOD range with a variety of new colours. The TePe GOOD range includes the Regular Soft Toothbrush, the UK's first plantbased plastic toothbrush, which was awarded Product of the Year 2019 by The Dental Guide in The Mail on Sunday.

Also, in the range is the TePe GOOD Compact toothbrush, TePe GOOD Mini Extra Soft toothbrush, TePe GOOD Mini Flosser 36-pack and TePe GOOD Tongue Cleaner, the UK's first plant based-plastic tongue cleaner.

To date, the range has only been available in green, highlighting TePe's
Patients can also benefit from the CPS Prime interdental brush with its reusable handle and replaceable brush head to ensure minimal plastic waste.

Recommend Curaprox products with confidence knowing they are innovatively designed to facilitate a gentle but effective clean - even in hard-to-reach areas.

For more information call 01480862084 , emailinfo@curaprox.co.uk or visitwww. curaprox.co.uk.

\section{Ultimate temporary}

\section{seal}

Where temporary fillings are needed for inlay/only preparations and endodontic applications, a reliable material is essential to protecting against the infiltration of bacteria and ensuring the patient continues to have full oral function.

For double protection try COLTENE's DuoTEMP temporary filling material with high marginal seal and dual-curing properties - it takes just 40 seconds to light cure DuoTEMP before it begins to self-cure on its own with saliva.

Once it's been applied, your patients will hardly know it's there thanks to the DuoTEMP's high surface hardness and natural tooth-like feeling, ensuring ultimate comfort until the material is replaced with a permanent solution.

The temporary restoration is very easy to apply and remove as well, which helps to reduce the time the patient needs to spend in the chair and maximises the patient experience.

To find out more visit www.coltene. com, emailinfo.uk@coltene.com or call 01444235486. rooftop solar panels at the Swedish factory in Malmö.

TePe will also be bringing back the TePe mini flosser as part of the GOOD range. The TePe GOOD Mini Flosser 36-pack - an innovative oral care product that is made from sugar cane, wood fibres and Teflon free floss. The plant based plastic packaging is also made from sugar cane.

For further information visit our sustainability pages at visit www.tepe.com/ uk.

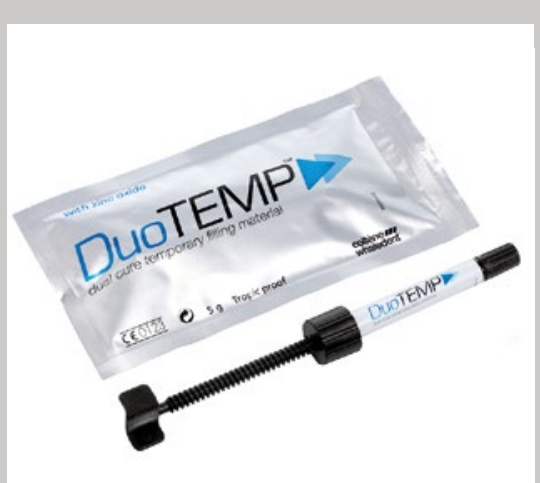

\title{
Access records anytime, anywhere
}

When you're working on a tricky case or want to access patient records on the move, it can be frustrating to not have the option of anytime access.

The new Care Management Platform from Carestream Dental not only allows professionals to access patient records on the go, but also offers a host of other benefits.
A software as a service, this cloudbased server is protected by state-ofthe-art security encryption and offers innovative business tools so that it is easier than ever to help your practice flourish.

For more information, contact Carestream Dental on 08001699692 or visit www.carestreamdental.co.uk.

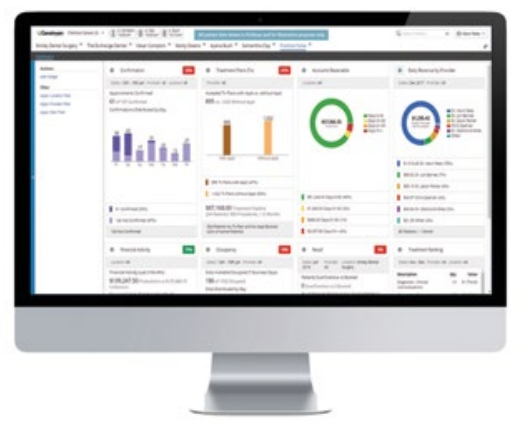

